\title{
Investigando con personas con dificultades de aprendizaje
}

\section{Researching with people with learning difficulties}

\section{Resumen}

El artículo muestra los orígenes de lo que Walmsley (2008) denomina «investigación inclusiva». Para comprender qué se entiende por investigación inclusiva tenemos que remontarnos a los debates epistemológicos sobre las metodologías cuantitativas y cualitativas, acontecidos en la década de los 90, en torno a la revista Disability \& Society. A partir de una síntesis de dichos debates, focalizados en el ámbito de la «discapacidad intelectual y del desarrollo", se exponen dos estrategias de colaboración con dicha población: a) una aproximación etnográfica (de trabajo grupal), y b) una aproximación biográfica (de trabajo individual).

A continuación se esboza un posible diseño de trabajo de campo que intenta superar el paradigma cualitativo "clásico» con el objetivo de incluir a dicho colectivo más allá del rol de "sujetos de la investigación». Para finalizar se recoge el debate sobre la accesibilidad de los resultados de la investigación a los participantes en dichas investigaciones, y con ello la necesaria innovación en el ámbito de las «devoluciones» de los resultados, cuando se trata de incluir a personas que presentan limitaciones para la comprensión del lenguaje abstracto oral y/o escrito.

\section{Palabras clave}

Modelo social, paradigma emancipador, investigación inclusiva, discapacidad intelectual o del desarrollo, personas con dificultades de aprendizaje, metodología participativa.

\begin{abstract}
The paper shows the origins of what Walmsley (2008) calls «inclusive research». To understand what is meant by inclusive research, we have to go back to the epistemological debate about quantitative and qualitative methodologies that took place in the 9os, around the journal Disability o Society. From a synthesis of these discussions, centered on the field of «intellectual and developmental disabilities», the paper presents two strategies for working with this population: a) an ethnographic approach (working group), and b) a biographical approach (of individual work). Then a possible fieldwork design is suggested trying to overcome the «classical» qualitative paradigm, with the aim of including people with disabilities beyond the role of «research subjects». The paper ends by presenting the debate on the accessibility of research results to participants in this type of research, as well as on the necessary innovation in the way this feedbak is provided, when it is to include people who have limitations for understanding abstract language, in oral and/or written form.
\end{abstract}

\section{Keywords}

Social model, emancipatory paradigm, inclusive research, intellectual or developmental disabilities, people with learning difficulties, participatory methodology.

\section{Borja González Luna \\ <bgonlun@gmail.com>}

Doctorando de la Facultad de Ciencias Políticas y Sociología de la Universidad Complutense de Madrid
Para citar:

González Luna, B. (2013):

"Investigando con personas con dificultades de aprendizaje". Revista Española de Discapacidad, I (2): 77-94.

<http://dx.doi.org/I0.5569/23405IO4.01.02.05>

Fecha de recepción: I2-I 2-2O I 2 Fecha de aceptación: O4-I I-2OI3 


\section{Introducción}

Para Morris (2008: 323-325), los investigadores sin discapacidad como aliados "cuando se ganan la vida mediante trabajos relacionados con el campo de la discapacidad, deberían preguntarse si pueden realizar una investigación que empodere a las personas con discapacidad y cómo podrían hacerlo”.

Este texto es el fruto de cuatro años de experiencia en el «sector de la discapacidad intelectual y del desarrollo» en España ${ }^{\mathrm{I}}$, trabajando con personas con dificultades de aprendizaje $\mathrm{e}^{2}$. La principal propuesta metodológica que se expone en el presente texto es que todo trabajo de campo que tenga como objetivo incluir como sujetos de la investigación, en un primer momento, y como "coinvestigadores, entrevistadores, consejeros, historiadores de vida o autobiógrafos», en un segundo momento, a población con dificultades de aprendizaje, debe partir de un conocimiento de las personas participantes en dicho contexto de investigación.

La experiencia acumulada en los últimos años me ha enseñado que la mayoría de personas con dificultades de aprendizaje que tienen dificultades en sus competencias comunicativas, tienen una mayor capacidad comprensiva que la que las personas que no les conocen les atribuyen. Es decir, mi prejuicio me decía que si una persona no se expresaba bien, no me iba a comprender bien. En cambio, he comprobado que muchas personas que tienen limitaciones comunicativas tienen más capacidades para comprenderme a mí, que yo a ellos.

I. Nota del Editor: Para más información sobre dicha experiencia, revisar la reseña biográfica del autor en el mismo número de esta revista.

2. Ante la falta de un auto-concepto proporcionado por el propio colectivo en España, voy a optar por usar para referirme de forma general al mismo el término que se han proporcionado los y las participantes del movimiento de autorepresentación en Inglaterra: "personas con dificultades de aprendizaje». Y utilizaré el término "discapacidad intelectual y del desarrollo» para referirme al «sector» de intervención social y estatal en su conjunto.
A partir de una lectura teórica de la experiencia empírica en el contexto anglosajón, se ha considerado necesario recoger las reflexiones, preguntas y críticas planteadas por James Walmsley (2008) con el objetivo de abrir un debate metodológico que apueste por dar voz y protagonismo a las personas con dificultades de aprendizaje desde una perspectiva «participativa».

Analizando los trabajos de Walmsley (2008: 373), son dos los horizontes empíricos que se nos propone superar desde la experiencia investigadora británica: "la mayoría de las investigaciones en el ámbito de la discapacidad intelectual y del desarrollo tienen como foco «cuestiones vivenciales» (cómo es el mundo para las personas con dificultades de aprendizaje) y «cuestiones de servicio» (cómo podemos crear mejores servicios para mejorar la vida de dichas personas)".

\section{Los orígenes del paradigma emancipador}

En la década de los 9o, en el marco de los desarrollos teóricos del modelo social en torno a la revista entonces llamada Disability, Hándicap and Society, actualmente conocida como Disability and Society, se desarrolló un debate epistemológico sobre si las investigaciones en discapacidad contribuían a cambiar «las condiciones de vida de los sujetos investigados». Según Oliver (2008), los defectos de la investigación, tanto cuantitativa como cualitativa, hay que buscarlos en lo que denomina «relaciones sociales de producción investigadora»:

"Estas relaciones se construyen sobre la base de una estricta distinción entre el investigador y el sujeto de la investigación; sobre la creencia de que son los investigadores los que poseen los conocimientos y habilidades propios de un especialista y de que son ellos los que deberían decidir sobre qué investigar y tener el control del proceso de producción investigadora en su totalidad" (Oliver, 2008: 300). 
Según Oliver (2008: 307 ) "la historia de la investigación en el ámbito de la discapacidad es, sin duda, una historia dominada por el paradigma positivista [...]. Esta dominación implica dos problemas importantes: en primer lugar, que la experiencia de la discapacidad se distorsionó profundamente; en segundo lugar, que los vínculos entre la investigación y el cambio social se tomaron como relativamente simplistas y racionales y se adoptó un enfoque relacionado con la ingeniería social para el proceso de diseño de nuevas políticas”. Para Oliver (2008), el paradigma positivista consideró la discapacidad como un problema individual y defendió, además, que la relación entre los resultados de las investigaciones y el cambio en las políticas públicas no era una cuestión problemática.

Por otro lado, desde la década de los 80 se hicieron algunos intentos de realizar investigaciones sobre discapacidad desde el marco del paradigma interpretativo. Las críticas a este paradigma se centraron en que este tipo de trabajo también fracasa a la hora de tener algún tipo de influencia determinante sobre los servicios para las personas con discapacidad y su calidad de vida. Según Oliver (2008: 308), "una vez más es posible identificar dos razones fundamentales por las que esto se produce; en primer lugar, este tipo de investigación no modifica la esencia de las relaciones sociales de producción investigadora. La segunda crítica proviene del modelo de formulación de políticas que presupone el paradigma interpretativo: lo que en general se denomina el modelo del iluminismo. Este modelo sostiene que no existe un vínculo directo y explícito entre la investigación y el diseño de nuevas políticas, sino que lo que hace la investigación es moldear el proceso de formulación de nuevas políticas".

Los defensores del modelo social, desengañados tanto con el paradigma positivista como con el interpretativo, formularon la necesidad de desarrollar un paradigma «emancipador» con el objetivo de hacer que la investigación en el ámbito de la discapacidad fuera más relevante para la vida de las personas que tienen una discapacidad y participan de dichos procesos.
Éste recibió diversos nombres: investigación crítica, praxis o investigación emancipadora ${ }^{3}$.

Los tres puntos clave sobre los que el «paradigma emancipador» se apoya son la «reciprocidad», el «beneficio» y el «empoderamiento». La reciprocidad hace referencia al intercambio personal que se da dentro de los procesos de investigación. En dichas relaciones, tanto el investigador como el investigado tienen mucho que aprender del otro si su mirada es abierta. En relación con el beneficio, las críticas estuvieron centradas, principalmente, en que eran los investigadores, con sus publicaciones y sus méritos, los que sacaban más provecho de dichas relaciones de reciprocidad. Es cierto que el investigador, en el curso de su carrera profesional, va acumulando méritos. Pero también es cierto que su trabajo no deja indiferente a la otra parte, y esas mismas relaciones de reciprocidad generan procesos de reflexividad que enriquecen a ambas partes. Los debates de los años 90 van a desplazar el objeto de investigación hacia lo que denominan "empoderamiento", es decir, hacia aquellos procesos que generan autonomía personal y colectiva.

La idea integradora que atraviesa el modelo de pensamiento social y la investigación emancipadora en discapacidad es su propósito transformador, es decir, la supresión de las barreras (materiales y sociales) y la promoción de la autonomía, tanto individual como colectiva, de las personas con discapacidad. Desde este punto de vista, la función del investigador es ayudar a facilitar la concreción de estos objetivos mediante el proceso de investigación. Desde el paradigma emancipador los investigadores deben aprender de qué manera poner sus conocimientos y aptitudes al servicio de los participantes del estudio.

\footnotetext{
3. A modo de aclaración, el término de paradigma «emancipador» o investigación "emancipadora» ha sido acuñado por las y los miembros de la comunidad científica a la que me estoy refiriendo. No me corresponde, como autor del artículo, juzgar si dicho término es el más apropiado. Mi intención es trasladar al lector las reflexiones epistemológicas acontecidas en la década de los 90 en Inglaterra y su contexto de influencia, y de forma más relevante el conjunto de investigaciones aplicadas que se referencian en las páginas siguientes.
} 
Figura 1. Investigación social y políticas sociales

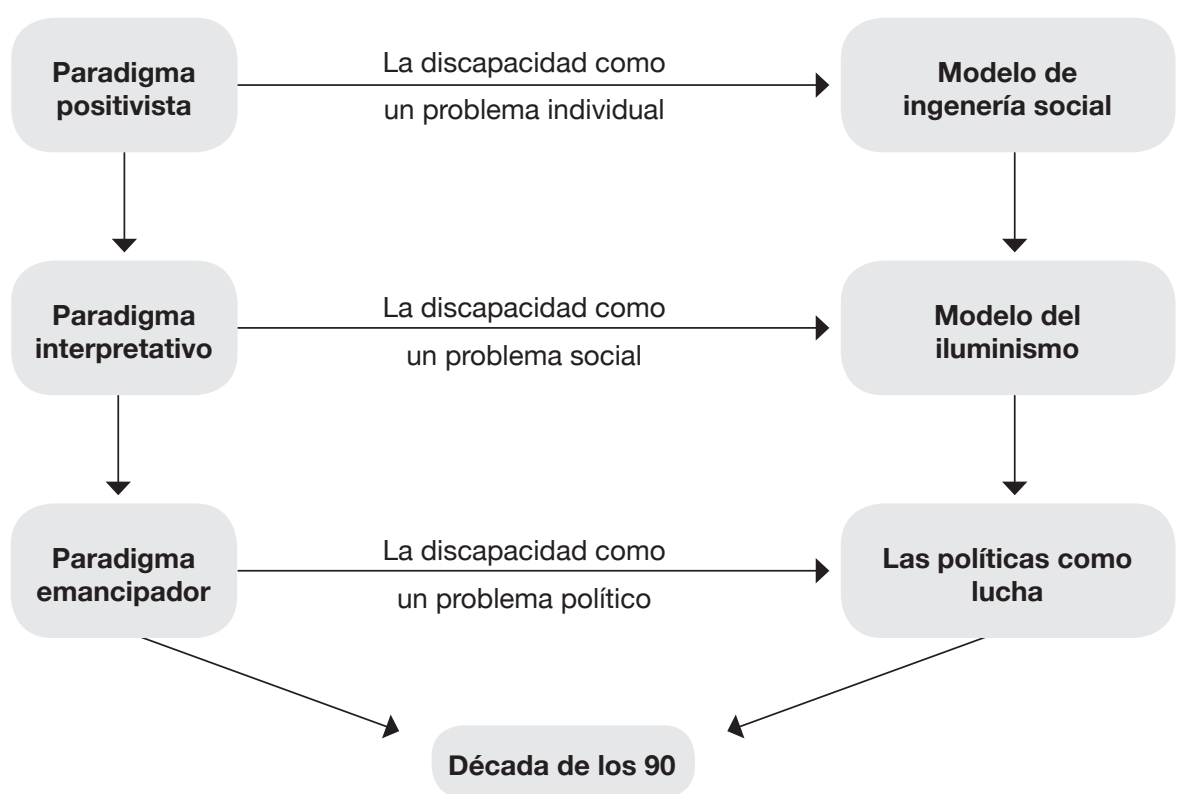

Fuente: Oliver (2008: 306).

Oliver (2008) plantea en una representación gráfica los diferentes paradigmas de investigación sociológica que han abordado el fenómeno de la discapacidad (Figura I).

\section{El paradigma emancipador en el ámbito} de la discapacidad intelectual

El desarrollo de las transformaciones culturales democratizadoras en las sociedades occidentales a partir de la década de los 60 y la aparición, a finales de dicha época, del actual movimiento por los derechos humanos en el ámbito de la discapacidad, provocó una trasformación en los estudios sobre personas con discapacidad. En la actualidad, dicho colectivo posee, según Gerber, (2008: 276) "una voz cada vez más autorizada en nuestros esfuerzos por conceptualizar las experiencias y el significado de tener impedimentos físicos y del desarrollo". Según este autor (ídem) "la negativa de una voz a las personas con discapacidad fue aún más determinante en el caso de aquellas clasificadas como retrasadas mentales”. Desde que Bogdan y Taylor (I976) publicaron "The judged, not the judges: an insider's view of mental retardation", que consistía principalmente en el testimonio oral de un hombre diagnosticado como retrasado mental, y desafiaron a los lectores a concederle la autoridad para que hablara sobre cómo entendía su propia vida, los investigadores buscaron cada vez más que las personas con dificultades de aprendizaje hablaran por sí mismas.

En palabras de Gerber (2008: 284):

"Antes de la década de los sesenta, los psicólogos eran los que dominaban los estudios sobre retraso mental y quienes, tal como señalaron Manion y Bersani (I987), disfrazaban sus suposiciones normativas con 
el lenguaje y los métodos de la ciencia. Los antropólogos como Edgerton (I967,I979), y aquellos especialistas en retraso mental como Bogdan y Taylor ( I976) que adoptaron métodos antropológicos, trajeron perspectivas nuevas y humanistas al estudio de esta condición. Mediante el uso de los métodos cualitativos de la etnografía (observación del participante, descripción narrativa y entrevistas en profundidad) intentaron presentar al individuo con retraso mental de manera empática y desde su propio punto de vista".

Para James Walmsley (2008), actualmente las investigaciones que incluyen a las personas con dificultades de aprendizaje son bastante comunes gracias al impulso que recibieron de parte de ideologías como la «normalización» y el «modelo social de discapacidad». Como ya se ha comentado anteriormente, el desarrollo de la investigación inclusiva en el ámbito de la discapacidad intelectual fue una característica específica de finales del siglo XX. No existen pruebas de que, antes del trabajo de Robert Edgerton en la década de los sesenta, alguien haya intentado acceder a las voces de las personas con dificultades de aprendizaje o representarlas en el campo de la investigación. Según Walmsley (2008: 360) "este campo estuvo dominado por la eugenesia, la psicología, los estudios educacionales y las investigaciones clínicas donde a las personas con dificultades de aprendizaje se las examinó, contó, observó, analizó, describió y, con frecuencia, se les diagnóstico una patología, pero nunca les consultaron sobre sus puntos de vista" ${ }^{4}$.

Walmsley (2008) utiliza el término «investigación inclusiva» para referirse a un número de enfoques de la investigación que tradicionalmente se calificaron como «participativos» o «emancipadores»; en términos generales, se aplica a investigaciones

4. Para un conocimiento más detallado de la aproximación antropológica a la discapacidad intelectual recomendamos al lector/a el texto de Guerrero Muñoz, J. (20IO): "La discapacidad intelectual en el contexto de la investigación etnográfica: rutas y enclaves”, Gazeta de Antropología, 26 (2), artículo 37 (en línea) <http://hdl.handle.net/I048I/6738>. en las que las personas con dificultades de aprendizaje se involucran más allá del rol de simples sujetos de estudio o participantes. En estas investigaciones, según Walmsley (2008: 360 ), "las personas con dificultades de aprendizaje asumieron diversos papeles, como coinvestigadores (March, I997), entrevistadores (Williams, I999), consejeros (Atkinson y Williams, I990; Stalker, I998), historiadores de vida de personas (Rolph, 2000) y autobiógrafos (Atkinson y Walmsley, I999)".

Sin embargo, en el ámbito de la discapacidad intelectual, Riddell et al. (I998) plantearon los límites de los principales criterios de la investigación emancipadora:

"Intentar cumplir con los tres criterios (de la investigación emancipadora) en la investigación acción con personas con discapacidad física es imaginable... intentar cumplir estos criterios con... personas con dificultades de aprendizaje es más complejo. La pericia del investigador... no se puede transmitir a un grupo de personas con impedimentos cognitivos; la participación de personas... podría estar limitada de manera similar; los modelos actuales... sugieren que las influencias tanto hacia las etapas triviales como a las realizadas por profesionales: son difíciles de resistir" (Riddell et al., I998: 8I-82)

A partir de estas experiencias investigadoras, Walmsley (2008) va a plantear que hay diversos temas que se tratan en la bibliografía sobre «investigación emancipadora» que todavía tienen que plantearse en el ámbito de la discapacidad intelectual y del desarrollo. Los temas que requieren de una mayor experiencia investigadora, de un mayor diálogo interdisciplinar y de una necesaria reflexión metodológica son:

- Quién debe llevar a cabo la investigación.

- Tema de investigación y enfoque.

- La investigación como apoyo activo.

- La accesibilidad de los resultados de la investigación.

5. Citado por Walmsley (2008: 37I). 
En consonancia con lo planteado, a continuación se desarrolla una propuesta metodológica que quiere integrar a personas con dificultades de aprendizaje dentro de procesos de investigación con el objetivo de que sean ellos y ellas, en primer lugar, los que definan los objetos de investigación. A partir de ahí, el propio contexto de investigación será el que definirá las posibilidades que las ciencias sociales nos permiten desarrollar. Teniendo presente como condición de partida que las diferentes disciplinas se construyen principalmente a través del lenguaje y el pensamiento abstracto.

\section{La aproximación etnográfica}

La experiencia en el campo de la discapacidad intelectual y del desarrollo me ha enseñado que si se quieren asumir las premisas del paradigma emancipador, todo diseño de investigación debe comenzar por conocer a los participantes del proyecto que se vaya a plantear. En relación con esta propuesta propongo plantear una metodología etnográfica de acercamiento y conocimiento de la población que «quiere» formar parte de la investigación de forma «voluntaria».

En relación con el tema de investigación, parto de la experiencia que dice que una vez que se ha empezado a conocer y conformar al grupo de trabajo con el que se va a trabajar, dicho grupo, en las diferentes conversaciones informales (con ellos y ellas, y sus redes más cercanas) van a proponer diferentes temas de investigación de gran relevancia. El primer paso será, a través de dinámicas participativas, relacionar dichos temas con el enfoque teórico del modelo social de la discapacidad. Si se quiere partir de dicho enfoque, pues como algunos autores y autoras (Morris 2008; Barnes 2008) han planteado, no existe la obligación de asumir el modelo social como un marco teórico imprescindible.

El objetivo de esta propuesta es compartir una representación gráfica y visual de las diferentes representaciones teóricas de partida con los miembros del «grupo conformado» ${ }^{6}$ con el fin de enseñarles a organizar la información que se va ir recogiendo con las diferentes dinámicas investigadoras (observaciones, entrevistas, descripciones, auto-narraciones, etc.). Se trata de dotar a los participantes de referentes comunes para que les sirvan de apoyo para comprender los fines del trabajo de campo y como punto de partida para analizar los materiales del trabajo de campo ${ }^{7}$.

La dinámica de trabajo que se propone es grupal y el investigador desempeña un rol de apoyo activo (donde se tendrá que valorar la presencia o no de los habituales apoyos técnicos y sus redes más cercanas). Aquí estarán presentes los interrogantes que plantea Walmsley (2008), es decir: cuando el grupo tome una decisión contraria al criterio técnico o familiar ¿cuál debe ser el rol del «investigador»?. Si nos apoyamos en la experiencia de la investigaciónacción-participación (IAP), el investigador no debería ser más que un «dinamizador» que pone su conocimiento en poder de los sujetos de la investigación para generar un proceso de reflexividad que tenga como objetivo el empoderamiento de dichos sujetos, como proceso de autorresponsabilidad y no de imposición moralizadora. A fin de cuentas, el investigador, más tarde o más temprano, abandonará el campo de trabajo. Desde la experiencia británica, Walmsley (2008) nos pone el ejemplo de Booth y Booth (I994: 24):

"Nuestro compromiso hacia los padres (con dificultades de aprendizaje) siempre estuvo por encima de los intereses de la investigación cada vez que los dos parecían dirigirse en direcciones opuestas."

Lo que se defiende es que la etnografía posibilita, como proceso dialógico, reflexivo

6. No todas ni todos los participantes tienen por qué finalizar el proceso de investigación. Algunos pueden abandonar el grupo si lo prefieren; otros u otras podrían entrar por curiosidad.

7. El diseño dependerá de las dinámicas que se desarrollen. Lo que puede llegar a negar la palabra, pero no otras vías de comunicación. 
y constructivo, responder a los principales criterios de la investigación emancipadora. Esta es una tesis que tomo de Davis (2008: 343), "la metodología etnográfica puede responder a los tres puntos clave sobre los que el paradigma emancipador debe basarse: la reciprocidad, el beneficio y el empoderamiento":

I. Las personas con discapacidad deben controlar el proceso de investigación en lugar de solamente participar en él. La etnografía permite que el investigador transmita parte de sus conocimientos a un grupo motor de personas con dificultades de aprendizaje que pueden llegar a adoptar alguno de los roles planteados por la «investigación inclusiva».

2. El investigador pasa de ser el intérprete "experto» del mundo a ser el servidor de las personas con discapacidad al poner sus habilidades a disposición de ellas. La etnografía, en cuanto intercambio cultural, fomenta los objetivos de la investigación emancipadora porque requiere que el investigador sea un participante reflexivo del proceso. Esto altera las relaciones cotidianas de la investigación, ya que otorga al etnógrafo posibilidades incontables para que ofrezca a sus sujetos de estudio la oportunidad de cuestionar su análisis.

3. Son las personas con discapacidad las que deben formular la pregunta que guiará la investigación. Al intercambiar interpretaciones sobre un mismo acontecimiento, el etnógrafo permite que las ideas de los participantes moldeen las etapas siguientes del estudio etnográfico (preguntas a realizar, situaciones a observar o experimentar, papeles a adoptar, etc.).

4. Y, lo que es fundamental, controlar los recursos. Aquí es donde la investigación emancipadora e inclusiva encuentra más dificultades, porque la mayoría de entidades del sector de la discapacidad intelectual están gestionadas por familiares de éstas o por profesionales que realizan tareas de gestión. Esto no impide que los recursos obtenidos por una entidad o por un grupo de investigación sean puestos a disposición del grupo motor planteado. Hasta la fecha no se conoce ninguna investigación de este carácter en España.

Otra reflexión propuesta por Walmsley (2008: 273) que resulta importante subraya que "hubo poco debate sobre qué personas con dificultades de aprendizaje pueden controlar la investigación", principalmente, cuando se trata de incluir a las personas que presentan impedimentos más severos y profundos. Y es en relación con esta población, es decir, la que presenta impedimentos para la comprensión del lenguaje y la comunicación de ideas, donde nos parece que la etnografía es la herramienta más adecuada para los objetivos planteados -en conjunción con las metodologías biográficas, que es la propuesta que se presenta en el siguiente apartado-.

Walmsley (2008: 375) va a sugerir que, “debido al desequilibrio de poder constante entre las personas con dificultades de aprendizaje y sus aliados, la postura de la investigación en cuanto apoyo activo es menos sencilla de lo que sugieren los debates en la bibliografía sobre estudios en discapacidad". Su argumento es que "aunque las personas con discapacidad pueden realizar sus debates sin hacer referencia a los miembros de los grupos opresores $-\mathrm{y}$ lo hacen-, las personas con dificultades de aprendizaje todavía dependen de un enfoque de trabajo en equipo, y son pocos los que pueden sostener que, sin ese enfoque, las personas con dificultades de aprendizaje se encontrarían en una posición que les permitiera investigar y publicar sus resultados" (Walmsley, 2008: 375 ).

Según Walmsley (2008), el investigador se ve obligado, en ciertas ocasiones, a desempeñar el «rol de la defensa ciudadana» tal y como ha sido formulado por Butler Carr y Sullivan:

"Ocurre cuando una persona que está muy vinculada con las redes de personas y asociaciones que integran la vida comunitaria y es independiente de los servicios, desea, sin recibir una remuneración a cambio, crear una 
relación con una persona que corre peligro de ser excluida socialmente, comprender, responder y representar sus intereses como si fueran los propios, de manera tal que presenta las aptitudes y preocupaciones de su compañero en los círculos de la vida comunitaria normal" (Citado en Williams, I990: I02).

La síntesis del debate es que existen posibilidades de cumplir los criterios planteados por el programa de investigación emancipador para una parte del colectivo que puede representarse. La pregunta metodológica a responder es: ¿quién debe representar a «las personas con dificultades de aprendizaje que presentan impedimentos para representarse»?. Esta es una de las preguntas teóricas que deben responder los proyectos de investigación que tengan como objetivo la inclusión de las personas que presentan mayores hándicaps ${ }^{8}$ para participar en las «relaciones sociales de producción investigadora».

\section{La aproximación biográfica}

La propuesta que se presenta a continuación se puede tomar como complemento de la anterior o como una estrategia diferente que buscaría conformar una muestra de personas con dificultades de aprendizaje que podrían ser usuarias de diferentes servicios y entidades. Lo que se propone es sustituir el modelo de trabajo grupal por una estrategia de trabajo individual. Esta propuesta podría integrar, en una fase posterior, la conformación de un grupo de trabajo a partir de los contactos individuales preliminares donde el investigador conocería

8. Entiendo por «hándicaps» cualquier situación que obligue al investigador o al equipo de investigación a modificar, adaptar o reinventar las habituales prácticas, en sentido amplio, de las ciencias sociales. Si el conjunto del colectivo exige de dicha modulación, por decirlo de alguna manera, de las fases y de dichas técnicas es una hipótesis que yo no he verificado empíricamente y que puede llegar a ser la expresión de un prejuicio. a las personas que desean participar en el proyecto 9 .

En relación con la justificación metodológica, por su carácter de encuentro personal con la persona, su red familiar, social e institucional, la metodología biográfica mantiene las mismas ventajas que anteriormente se han planteado en relación con el trabajo etnográfico e, incluso, la historia de vida podría ser la mejor estrategia cuando se trata de acceder y devolver los resultados de una investigación a una persona con impedimentos para la comunicación. Un ejemplo gráfico de lo que se defiende lo constituye el documental “¿Qué tienes debajo del sombrero?", donde se narra la historia de vida de una persona sordomuda y con síndrome de Down que a lo largo de su vida ha desarrollado formas de comunicación «no verbal» con su red familiar, social e institucional. A través de metodologías audiovisuales, el documental recoge un proceso de participación social a través de la creación artística. ${ }^{\text {ㅇ }}$

\subsection{Ejemplos prácticos}

A continuación se exponen tres experiencias investigadoras ${ }^{\mathrm{II}}$ que pueden servir de ejemplo de las propuestas metodológicas que se han realizado. En primer lugar, se pone como ejemplo la experiencia de dos investigadoras australianas, Welsby y Horsfall, que publicaron un artículo en Disability and Society, en

\footnotetext{
9. Una vez más, son propuestas de acercamiento que requieren de experiencias de investigación. De la misma forma, el trabajo grupal presentado anteriormente se puede complementar con entrevistas biográficas que ayuden al equipo de investigación a conocer mejor a las personas integrantes del grupo de trabajo.

Io. En la base de datos documental del «Servicio de Información sobre Discapacidad» y del «Centro Español de Documentación sobre Discapacidad», podemos encontrar trabajos realizados en otras disciplinas de gran utilidad para la sociología: Ballesta (20I I), Díaz et al. (200I), Flores y Romero (2008), Gallego (2008), Muñoz et al. (2009), Ochoa de Eribe (I994).

I . La experiencia de los grupos Autogestores de FEAPS en España siempre me ha parecido un espacio muy apropiado por el que empezar a realizar investigaciones inclusivas con personas con dificultades de aprendizaje; sin olvidar, una vez más, a aquellas personas que presentan mayores hándicaps para la comunicación y comprensión lingüística.
} 
Diciembre del 20I I, con el nombre: "Prácticas cotidianas de exclusión/inclusión: Mujeres que tienen una discapacidad intelectual hablando por sí mismas” I2. Principalmente nos centramos en la metodología desarrollada. Según Welsby y Horsfall (20II):

"No todos los actores sociales encuentran en la forma más usual de entrevista e historias orales la mejor manera de contar sus historias (ver McCarthy 1998; Maynard 2002). Esto debe ser el resultado de no haberse preguntado nunca, y por tanto no haber tenido la oportunidad de descubrir sus habilidades. $\mathrm{Y}$, a veces, para la gente es un reto "físico» e «intelectual» decir lo que les preocupa y porqué. Las entrevistas priorizan la palabra hablada, y con esto a aquellas personas que puedan hablar de un modo particular. Sin embargo, hacer uso de múltiples formatos de texto puede habilitar que la gente hable por sí misma, definiendo sus vidas con sus propias palabras.

[...] Reconociendo las limitaciones potenciales de confiar únicamente en entrevistas basadas en la narración, descubrimos un formato de investigación creativo basado en el arte, no de modo terapéutico sino para usar procesos artísticos para crear espacios discursivos que permitirían a las mujeres hablar si querían y cómo querían",(Welsby y Horsfall, 20I I: 797798). ${ }^{\text {13 }}$

A partir de esta hipótesis de trabajo, Welsby y Horsfall realizaron una investigación con cinco mujeres, de entre 28 y 52 años, que vivían en los suburbios de Sydney. En el momento de la investigación, estaban recibiendo una pensión de apoyo a las personas con discapacidad y trabajaban media jornada tanto en el Servicio de Empleo como en empleos ordinarios. Dos de

I2. En dicho artículo se cita una bibliografía muy interesante sobre «investigación inclusiva» para los lectores y las lectoras más interesadas: Welsby 20I0; Roets, Reinaart, y Van Hove 2008; Roets y Van Hove 2003; Atkinson, McCarthy, y Walmsley 2000, Bjornsdóttir y Svensdóttir 2008; Susinos 2007; McVilley et al., 2006; Grundy, McGinn, y Pollon 2005; Hall 2004; Walmsley y Johnson 2003.

I3. Ver Welsby (2010) para más detalles del proceso basado en el arte. las mujeres vivían en casas de acogida, una con sus padres y las dos restantes vivían de forma independiente en la comunidad.

Se hicieron cinco talleres cada dos semanas y fueron coordinados por un profesor de arte experimentado. Se proveyó a las participantes con una serie de medios y técnicas artísticas que podían elegir para trabajar. El objetivo era facilitar un espacio tan abierto como fuese posible para que cada mujer pudiese ejercitar cierto nivel de elección y autodeterminación. La temática, incluyendo el trabajo, la amistad, la identidad y la vida cotidiana, fueron sugeridas por las mujeres en el primer taller de formación sobre la técnica artística. Entre talleres, se llevaron a cabo entrevistas personales semiestructuradas. Las entrevistas se hicieron donde los participantes elegían, normalmente en una cafetería. Las entrevistas duraban aproximadamente una hora. Las anotaciones de campo, fotografías y transcripciones de las entrevistas sirvieron como datos, que fueron analizados por temas en un proceso dirigido que permitía a los temas emerger. Las creaciones artísticas no eran datos primarios. En vez de eso, la actividad artística era el vehículo para que las mujeres contasen historias sobre sus vidas. Según Welsby y Horsfall (20I I: 798), “los talleres fueron un éxito para hacer hablar a las mujeres. Cuando trabajaban individualmente, alrededor de una gran mesa, las historias se compartían y discutían y las conversaciones fluían de tema en tema, creando una serie de introspecciones colaborativas que estimulaban para contar historias personales y compartir experiencias".

Para Welsby y Horsfall (201 I: 797) “investigar sobre las experiencias vividas por gente con discapacidad intelectual, y mucho más investigar con ellos, es limitado”. Estas autoras, a través de un método de trabajo innovador, que pretendía superar las limitaciones de la práctica de la entrevista semi-estructurada, exploraron las nociones de inclusión/exclusión desde sus puntos de vista e incluyeron a dichas personas en el "proceso de investigación", a través de un taller de creación artística. Y en «algún grado» en el texto del artículo cuando 
hablan, a veces, junto a ellas: "como autoras, en cualquier caso, reconocemos que nuestras voces e interpretaciones son dominantes pues nos pertenece la autoría. Incluso aunque hayamos incluido las palabras de las mujeres en este artículo, somos nosotras -Janette y Debbielas que hemos escrito este artículo" (Welsby y Horsfall, 20I I: 797).

En este ejemplo podemos observar cómo algunos criterios del paradigma emancipador o inclusivo no se llegan a alcanzar. Verdaderamente, no es eso lo que más nos debería preocupar ni importar. Lo que es de interés destacar es que se trata de una investigación que está dando soluciones a problemas reales. Ante una población objeto, ante la insuficiencia y las limitaciones de unas prácticas de investigación, en este caso la entrevista cualitativa, las autoras desarrollan una estrategia de colaboración de carácter etnográfico, a través de un taller de creación artística, para obtener un espacio de convivencia y confianza en el que los sujetos de la investigación pudieron apropiarse «de las relaciones sociales de producción investigadora». Ellas mismas son conscientes de las limitaciones de su trabajo y reconocen ser dominantes en las interpretaciones, al ser ellas las que han escrito el artículo.

En segundo lugar, la experiencia que voy a narrar, como ejemplo práctico, es mi trabajo de fin de máster. En un principio, el objetivo era realizar entrevistas biográficas a personas con dificultades de aprendizaje, sin la mediación de profesionales ni familiares. Ante la falta de experiencia, en la relación directa con el colectivo, mi tutor de proyecto me recomendó que realizara un voluntariado en una entidad para conocer de cerca la realidad de las personas con dificultades de aprendizaje. A partir de ahí surgió la idea de realizar, en primer lugar, un trabajo etnográfico como estrategia de acercamiento y conocimiento de las personas participantes en una asociación de ocio y tiempo libre que, en segundo lugar, iba a entrevistar.

Finalmente, este voluntariado fue de 6 meses en una asociación de ocio y tiempo libre del municipio de Fuenlabrada. Dentro de esta asociación, participé como jugador de baloncesto en un equipo integrado por personas con y sin discapacidad, que juega en una de las ligas regulares del municipio de Fuenlabrada (es el único equipo con personas con discapacidad de la liga). Y también participé como voluntario en las salidas de «ocio compartido» de los fines de semana.

Durante el trabajo de campo tuve la oportunidad de realizar 6 entrevistas biográficas en los domicilios familiares de los participantes del equipo de baloncesto que quisieron participar en el proyecto. En el marco del máster de innovaciones y aplicaciones, realicé todas las entrevistas con cámara de video con el objetivo de meta-analizar la validez del guión de la entrevista y la interacción verbal y no-verbal. En consonancia con las reflexiones de Welsby y Horsfall (20II) constaté que la entrevista cualitativa era «insuficiente» con dos hermanos diagnosticados con «x frágil». El contexto de convivencia de los entrenamientos, los partidos de baloncesto, las salidas de ocio, etc., me habían proporcionado muchísimos más momentos para conocerlos y obtener opiniones sobre los temas que a ellos les interesaban que el rígido guión planteado y mi rígida formación. Y algo más importante, para ellos era mucho más fácil hablar sobre un tema en el contexto en el que ese tema se estaba desarrollando (deporte, ocio, amistad, trabajo, amor, emociones, etc.) que abstraerse para hablar de temas que no tenían nada que ver con el momento presente como acontece en el curso de la entrevista-.

En el caso de los otros 4 entrevistados restantes, la entrevista funcionó bastante bien. Sus capacidades comunicativas y comprensivas les permitían seguir el guión y responder a la «mayoría» de las preguntas ${ }^{14}$. Otra cuestión, al hilo de estas reflexiones, es si el guión de dicha entrevista fue relevante para sus vidas y los temas que tratamos eran de su interés. Por

I4. Sería muy extenso detallar los temas que estaban excluidos de su realidad cotidiana, por ejemplo, no llegaban a comprender qué era un «movimiento social». Los resultados de dicho trabajo saldrán en un futuro artículo. 
lo general, todos se mostraron satisfechos de colaborar en el proyecto.

En relación con el uso de la cámara de video, se ha escrito mucho sobre la reactividad que genera el uso de dicha técnica. Sin embargo, en este caso la confianza que había alcanzado con ellos hizo que no se generara tanta reactividad. Aun así, el análisis «interaccional» desvela cierto control y ocultación de información importante para la investigación. Por ello, es de considerar, sin deslegitimar el uso de la entrevista en profundidad, el adecuado "uso combinado» de las metodologías etnográficas y biográficas cuando se pueda ${ }^{15}$.

El tercer ejemplo está relacionado con el uso de metodologías cuantitativas dentro de un proceso participativo. En 2002 apareció en Siglo Cero la traducción de un artículo de dos investigadores, Ward y Trigler, pertenecientes al Center for Human Development de la Universidad de Alaska. En el artículo, que tiene por título "Reflexiones sobre la investigación participativa con personas que tienen discapacidades en el desarrollo", los autores cuentan una experiencia investigadora con la organización "People First" ("Ante todo personas") donde el Comité Directivo de la Primera Conferencia Internacional les encargó que les ayudaran en el proceso de llevar a cabo una encuesta sobre "calidad de vida» durante dicha conferencia.

Según Ward y Trigler (2002: 46):

"Involucrar a las personas con discapacidades cognitivas en el análisis de los datos y en la fase de interpretación de la investigación supone un verdadero desafío. Si bien hay que dedicarle tiempo, el esfuerzo de incluir a estas personas en los procesos de codificación de

I 5. La distinción que hago entre entrevista semiestructurada y entrevista biográfica es que mientras la primera puede consistir en uno o dos encuentros con el o la entrevistada, la entrevista biográfica consiste en una relación más estrecha entre el investigador y él o la investigada, y suele prolongarse más en el tiempo. También podría hacerse una diferenciación por el tipo de guión a desarrollar, y no tanto por el tipo de encuentros. Pero, en definitiva, aquí la distinción que se está remarcando es la de un mayor trato personal y conocimiento por ambas partes. datos y de análisis es necesario para que se dé un verdadero enfoque participativo. Resulta más difícil explicar el significado de pruebas estadísticas tipo test. Nuestros intentos por incluir autodefensores en la interpretación fracasaron en gran medida debido a la dificultad que les suponía extrapolar datos más allá de su propia experiencia personal, con el fin de entender el significado de los datos para un grupo representativo".

Para hacer posible una mayor implicación y control del proyecto, el Comité Directivo nombró un subcomité de cinco autodefensores para que desarrollara el cuestionario. Según Ward y Trigler (2002: 46): "los debates sobre qué cuestiones eran necesarias, apropiadas y útiles fueron animados e interesantes. Los miembros del subcomité compartieron historias de sus experiencias para ilustrar porqué una pregunta era necesaria o porqué una pregunta podría incomodar a algún encuestado”. El subcomité tomó las decisiones finales sobre la manera en que debería formularse las preguntas, qué preguntas incluir en la encuesta y la escala de puntuación. Según Ward y Trigler (2002: 46), "en nuestro deseo de ceder el control sobre el cuestionario a los autodefensores, creamos problemas metodológicos que de alguna forma comprometieron los resultados. Hemos aprendido que la clarificación de roles al principio del proceso, la identificación de áreas exclusivas para expertos y el establecimiento de las líneas maestras del proceso que desarrollará el equipo mediatizaba los temas del poder y del control".

Otro tema puesto en relevancia por estos autores es el tiempo de dedicación, tanto para investigadores como para interesados, que suponen estas metodologías. Según Ward y Trigler (2002: 47):

"Es cierto que los interesados al iniciar el estudio estaban muy motivados, pero la cantidad de tiempo requerida fue subestimada por todos los involucrados. El diseño original pretendía que los autodefensores estuvieran activamente involucrados en el desarrollo del cuestionario, en el estudio piloto y en el 
proceso de recolección de datos. Por desgracia la dedicación de los autodefensores se quedó muy por debajo de los objetivos iníciales".

El desarrollo del cuestionario les llevó más de cuatro meses. En ese tiempo, la asistencia a los encuentros de los miembros del Subcomité varió. Únicamente dos miembros fueron constantes en su asistencia y jugaron el papel más importante en el desarrollo del cuestionario. El cuestionario fue probado de forma experimental en una posterior asamblea de People First y ni uno solo miembro del Subcomité pudo asistir debido a problemas irresolubles de agenda. Por lo tanto, un investigador fue el encargado de dirigir esta prueba piloto. A medida que se iba acercando la fecha de la conferencia, se hizo más patente que la disponibilidad de los autodefensores para llevar adelante las entrevistas y recoger los datos en la conferencia era más bien limitada. Ellos se involucraron más en otros aspectos relacionados con la conferencia. Por lo tanto, personas sin discapacidad (estudiantes) terminaron dirigiendo la mayoría de las entrevistas.

Para Ward y Trigler (2002: 47), "si el proyecto hubiera tenido menor alcance y hubiera estado terminado en el plazo de unos pocos meses quizás el nivel de interés y la respuesta hacia los resultados alcanzados hubiera sido mayor”. Entre su aprendizaje, algo a destacar es "ganar capacidad y competencia en la investigación participativa requiere práctica y experiencia” (Ídem). También resulta muy interesante una de sus conclusiones finales: "es necesario que se realice mucho más trabajo para refinar los procesos mediante los cuales los individuos con discapacidades en el desarrollo sean participantes activos en los procesos de investigación que afectan a sus vidas" (Ídem).

Con este último ejemplo práctico se quería mostrar que también existen posibilidades de aplicar este diseño con metodologías cuantitativas y que la apuesta por un trabajo etnográfico y biográfico no representa una negación en la utilización de escalas de medición u otras técnicas. Si bien la investigación inclusiva o participativa supone un reto en el que no siempre se alcanzan los objetivos de partida, estoy de acuerdo con Ward y Trigler (2002) en que la implicación de los participantes, más allá del rol de sujetos, contribuye enormemente a la riqueza y la calidad de la investigación, a pesar de los problemas metodológicos.

\section{Más allá de las clásicas prácticas del paradigma cualitativo $^{16}$}

Las propuestas anteriores, tanto la aproximación etnográfica de trabajo grupal como la aproximación biográfica de trabajo individual, no son nada nuevo y en sí mismas no representan ningún desafío al reto planteado por la experiencia empírica anglosajona. Es oportuno recordar las palabras de Walmsley (2008: 373) cuando plantea que "la mayoría de las investigaciones en el ámbito de la discapacidad intelectual tienen como foco «cuestiones vivenciales» (cómo es el mundo para las personas con dificultades de aprendizaje) y «cuestiones de servicio» (cómo podemos crear mejores servicios para mejorar la vida de las personas)".

Las siguientes estrategias tienen como objetivo «superar» el enfoque cualitativo clásico que asume que proporcionar relatos fieles sobre las experiencias individuales es «suficiente» $\mathrm{y}$ plantea que la investigación debería ir más allá de las nociones de la «experiencia individual» para comprender la «experiencia colectiva» en el conjunto de la «estructura social», en un «territorio concreto ${ }^{17}$ :

16. La Oficina para Asuntos de Discapacidad del Gobierno Inglés, Office for Disability Issues (<http://odi.dwp.gov.uk/>), editó en 20 I I una guía con recomendaciones para incluir en los procesos de investigación a las personas con discapacidad: $<$ http://odi.dwp.gov.uk/docs/res/research/involving-disabledpeople-in-social-research.pdf $>$.

I7. La idea no es que este planteamiento metodológico responda a preguntas que se corresponden con análisis distributivos o estructurales de la «estructura social», más bien estos estudios se complementarían con los trabajos realizados por otros compañeros y compañeras en ese nivel de análisis. 
a. Del acercamiento etnográfico a la entrevista focalizada:

Lo que se plantea es que los miembros del equipo de trabajo podrían realizar entrevistas focalizadas, es decir, enfocadas al tema de investigación seleccionado y a las personas consideradas más relevantes de su red familiar, social e institucional. En la medida de las capacidades de los participantes estas entrevistas podrían ser más simples o más complejas, más breves o más extensas.

\section{b. Del acercamiento biográfico a la etnografía} focalizada:

En la segunda propuesta, lo que se plantea es que a partir de la conformación de una muestra de personas decididas a participar en el proyecto de investigación, y a través de unas primeras sesiones biográficas, se iría construyendo el tema de interés para las personas participantes. A partir de la definición de dicho tema se definirían los campos de observación más relevantes donde la persona con discapacidad realizaría una etnografía focalizada del tema de investigación seleccionado.

Creo que es obvio que el diseño que estoy planteando no es una cuestión que se resuelva teóricamente y sobre el papel. Por tanto, lo que se hace necesario es tener experiencias investigadoras, llámeselas «inclusivas» $\mathrm{O}$ «participativas», que tengan como objetivos responder a los interrogantes que se han ido desarrollando a lo largo de este artículo. De forma resumida, una vez más, considero que el uso combinado de las metodologías "etnográfica» $\mathrm{y}$ «biográfica» es un buen punto de partida. La decisión de partir de un plan de trabajo en grupo o individual, en principio, dependerá del grupo de investigación. También van a influir, como ya se ha comentado, las singularidades de las personas participantes -sobre todo en lo relacionado con la comunicación oral y escrita y con la comprensión de ideas-.

Adelantándome a una crítica histórica a los estudios microsociológicos, podemos decir junto con Bertaux (I997) que el funcionamiento de un sector de actividad como es el de la discapacidad intelectual y del desarrollo, puede engendrar una variedad más o menos grande de tipos de microcosmos; estudiar uno solo conduciría a generalizar abusivamente las características propias de un tipo social. Para evitar este error, hace falta multiplicar los campos de observación y compararlos. Por lo tanto, no es indispensable que este trabajo comparativo sea realizado por el mismo investigador: la investigación es una empresa colectiva y, en principio, acumulativa, a la que cada investigación aporta su propia contribución.

En España, desde comienzos del año 2000 el número de investigaciones que han ido dando voz a personas con discapacidad intelectual o del desarrollo ha ido en aumento ${ }^{18}$. Entre estas investigaciones, destacan temas de estudio clásicos, relacionados con la integración escolar, el empleo ordinario o el envejecimiento, y nuevos temas emergentes, como la autopercepción, el ocio, la salud, la calidad de vida, el empoderamiento. También se recogen algunos ejemplos de historias de vida contadas por familiares de personas que presentan algún impedimento para la comunicación oral. En algunas investigaciones la muestra estuvo formada por casos únicos y en otros por casos múltiples. La técnica cualitativa más utilizada es la «entrevista semi-estructurada» y, en menor medida, el «grupo de discusión». También, en triangulación o no con dichas técnicas, se ha contado con la opinión del colectivo a través del uso de escalas de percepción y/o valoración.

I 8. Una revisión bibliográfica nos muestra los siguientes ejemplos ordenados cronológicamente (cuyas referencias completas se muestran en la bibliografía): (Caton, S.,20I3), (Tamarit, J.y Espejo, L., 20I3), (Áriz, A., 20I2), (Badia, M., 20I 2), (Fernández-Cid Enríquez, M., 20I2), (Santamaría, M., 20I2), (Flores, N., 20I0), (Rodríguez, M. C., 20I0), (Rosa, L., 2008), (Verdugo, M. A. y Rodríguez, A., 2008), (Echeita, G. y Jiménez, D., 2007), (Leon, A., 2006), (Alomar, E. y Cabré, M., 2005), (Fullana, J., 2003). 


\section{La accesibilidad de los resultados de la} investigación

En último lugar voy a recoger los interrogantes propuestos por Walmsley (2008) en relación con la accesibilidad de los resultados de la investigación. Según esta autora (2008: 376), "hacer que la investigación sea accesible es, en teoría, esencial en lo que respecta a la investigación emancipadora; de no ser accesible, la totalidad de la iniciativa sería espuria”. En el contexto anglosajón, cada vez es más normal la producción de versiones accesibles del informe final que utilizan mecanismos, como el lenguaje simple, viñetas, símbolos, fotografías, ilustraciones y materiales audiovisuales, conocidos en nuestro contexto como textos de «lectura fácil».

En relación a la pregunta de cómo deben elaborarse dichos informes y qué objetivos deben cumplir, Walmsley (2008) nos expone el ejemplo del centro de investigación Norah Fry y la fundación Joseph Rowntree, los cuales han desarrollado un enfoque que llamaron "de los Hechos Simples” con el fin de difundir la información relacionada con la investigación. La complejidad está en que para que la investigación sea accesible se ha de evitar la formulación de teorías, a menos que ésta se realice a un nivel que las personas con dificultades de aprendizaje las puedan comprender.

La transmisión de resultados a través del lenguaje puede ser, para una parte del colectivo, una limitación. Por tanto, se hace necesario, si se quiere incluir a dicha población, una innovación metodológica en las formas de devolución de los resultados que vayan más allá del texto y la imagen. Por ejemplo, a través de dinámicas visuales y corporales, como puede ser el rolplaying, metodologías audiovisuales u otras con las que estén familiarizados los participantes, como por ejemplo los pictogramas.

En todo caso, es de gran relevancia establecer como principio metodológico que todo diseño de la investigación, como se viene planteando en relación al tema de la misma, enfoque, técnicas, etc., dependerá de un conocimiento previo de los y las participantes en el proyecto y de las problemáticas enunciadas por éstos y éstas, donde el investigador debe transformarse en una especie de socio-pedagogo, es decir, un experto en la transmisión de los conocimientos, habilidades y prácticas de las ciencias sociales desde la perspectiva participativa o inclusiva que aquí estoy defendiendo: con el objetivo de generar procesos de reflexividad, reciprocidad y empoderamiento, es decir, que los procesos sociales de investigación contribuyan a potenciar sus capacidades de autorrepresentación y autodeterminación -en la medida de las posibilidades de cada persona-.

\section{Conclusiones}

Las propuestas de acercamiento, conocimiento e inclusión de la población participante en el proyecto de investigación son unas propuestas «flexibles», que en el camino tomarán rumbos diferentes, conformando la experiencia investigadora a transmitir en futuros artículos y comunicaciones. Pero lo que Walmsley (2008) nos está sugiriendo es que no sólo debemos ser innovadores en nuestros planteamientos metodológicos sino en la definición de nuestros objetos de estudio. Y ahí la investigación inclusiva es muy clara: son los sujetos de la investigación los que deben definir los «nuevos objetos» hacia donde la investigación debe dirigir sus esfuerzos.

El sociólogo puede desempeñar el rol de metaanalizador de las «relaciones sociales de la producción investigadora» en el marco del paradigma emancipador, donde «la reflexividad, reciprocidad y empoderamiento» serían el objeto de investigación para el desarrollo de un programa de investigación que asuma el reto de incluir en dichos procesos a las personas con discapacidad. Es decir, se trata de ir acumulando experiencias, en diferentes investigaciones, sobre las variables que más influyen en los procesos de 
autorrepresentación y autodeterminación de los participantes.

Una de las principales líneas de «reflexión» metodológica son los procedimientos y limitaciones en la transmisión de las diferentes fases que componen una investigación: la definición del tema, la elaboración de las preguntas de investigación, la formación en las técnicas, el diseño metodológico, la recogida de los datos, el análisis de los materiales de campo, la elaboración de las conclusiones. Todo esto siempre en relación con los objetivos de la investigación y las demandas de los participantes en la misma.

Hay que considerar que tan contraproducente es hablar por el otro, como obligar a otro a hablar por sí mismo, es decir, la investigación inclusiva no tiene por qué obligar a los sujetos a ser partícipes de su problemática si ellos y ellas por sí mismos no la perciben como tal. En tal caso, el investigador, en su rol de apoyo activo, y desde una ética investigadora, sólo puede generar procesos de reflexividad. Y él mismo tendrá que reflexionar si desempeña el rol de la defensa ciudadana cuando, por ejemplo, se encuentre que se incumple la Convención Internacional sobre los Derechos de las Personas con Discapacidad.

Otra línea de desarrollo metodológico tiene que ver con la «traducción»y «comprensión» de los diferentes modelos teóricos que han definido la discapacidad, con el objetivo de dotar a las personas de las ideas necesarias para re-pensar sus vidas. Este es un aspecto importante si se quiere que las personas participantes en el proyecto alcancen la autonomía necesaria para elaborar sus propias propuestas teóricas según sus posibilidades. En el caso de que una persona o grupo de personas no puedan acceder a dichos modelos, habrá que atender a los cambios que el propio proceso de investigación genera en las vidas de dichas personas. Porque una propuesta teórica puede ser expresada, también, a través de una actitud, un gesto o un hecho.

La transmisión de prácticas sociológicas, en nuestra opinión, deben nutrirse de la experiencia de la Investigación-Acción-Participativa (IAP) en España ${ }^{19}$. Nos parece valioso considerar que dichas experiencias, en otros ámbitos de investigación, constituyen el punto de partida para una sociología de la discapacidad que pretenda asumir las premisas de la investigación emancipadora o inclusiva en nuestro contexto.

La accesibilidad y difusión de informes de investigación en el ámbito de la discapacidad intelectual implica innovaciones en la «devolución» de los resultados. No puede haber una planificación teórica en este tema sino que es necesario el conocimiento de la población participante y una alianza, para la construcción de dichas devoluciones, con las personas que mejor les conocen. Así como desarrollar estrategias de colaboración con otras disciplinas académicas especializadas en temas específicos como la comunicación alternativa, no verbal o artística.

En relación con la autoría del texto voy a volver a tener en consideración la opinión de Walmsley (2008: 375): "el hecho de que las personas con dificultades de aprendizaje necesiten aliados o defensores que les permitan realizar sus investigaciones implica que son extremadamente vulnerables a que otras personas tergiversen sus intereses. En una situación como esta, la investigación como apoyo activo pone en los hombros de las personas sin discapacidad la pesada carga de tener que actuar de manera desinteresada, ya que ellos son generalmente los encargados de plasmar los resultados en palabras”.

Resulta de relevancia, a partir de la lectura de los trabajos referenciados del contexto anglosajón, tener experiencias de investigación que nos ayuden a transmitir de forma más práctica y concreta los modos más adecuados de contribuir al cambio en las condiciones de vida

I9. En relación con la Investigación-Acción-Participación en España recomendamos al lector el capítulo escrito por Ortí Mata y Díaz Velázquez (20I2): “Claves teóricas y metodológicas para la Investigación Acción Participativa (IAP)", en Arroyo, M. y Sádaba, I. (Coords.): Metodologías de la Investigación Social. Técnicas innovadoras y sus aplicaciones. Madrid: Síntesis: I 53-I74. 
de las personas con discapacidad en el contexto español, al mismo tiempo que partimos del objetivo de superar las reflexiones, preguntas y críticas planteadas por Walmsley (2008) en relación con los estudios con población con discapacidad intelectual y del desarrollo.

Por último, la sociología no puede dejar en los márgenes a las personas que representan un mayor desafío a las técnicas de investigación que dicha disciplina ha desarrollado en el último siglo por presentar impedimentos para la comprensión y comunicación lingüística. Precisamente porque dicha población es la que permanece más invisible a los ojos de la opinión pública, porque es la que presenta los mayores grados de institucionalización, porque es la que se ve en mayor medida sometida a la medicalización y a los disciplinamientos del cuerpo, y porque cuando rechazan su situación vital a través de la «desobediencia» estos actos son clasificados como «trastornos de la conducta» $\mathrm{o}$ «disconductas», cuando no siempre lo son. 


\section{Referencias bibliográficas}

Alomar, E. y Cabré, M. (2005): "El trabajo de jóvenes con discapacidad intelectual en entornos normalizados". Revista Síndrome de Down, 22(4): II 8-I 24 .

Áriz, A. (20I2): La alegría muda de Mario. Pamplona: Sahats.

Badia, M. et al. (2012): "Participación y barreras percibidas en el ocio por los jóvenes y adultos con discapacidades del desarrollo", en Verdugo Alonso, M. A. (Dir.): Aplicación del paradigma de calidad de vida a la intervención con personas con discapacidad desde una perspectiva integral. Colección Investigación 7, Salamanca: Instituto Universitario de Integración: 83-94.

Ballesta, A.M. et al. (20I I): "El arte como un lenguaje posible en las personas con capacidades diversas”. Arte y Políticas de Identidad 4: I37-I 52.

Barnes, C. (2008): "La diferencia producida en una década. Reflexiones sobre la investigación emancipadora en discapacidad”. En Barton, L. (Comp.): Superar las Barreras de la Discapacidad. Madrid: Morata: 38I-395.

Bertaux, D. (I997): Histoires de Vie. Paris: Nathan.

Bogdan, R. y Taylor, S. (1976): “The judged, not the judges: an insider's view of mental retardation". American Psychologist,

3I: $47-52$.

Booth, T. (I998): "El sonido de las voces acalladas: cuestiones acerca del uso de los métodos narrativos con personas con dificultades de aprendizaje”, en Barton, L. (Comp.): Discapacidad y Sociedad. Madrid: Ed. Morata: 253-269.

Caton, S. et al. (2013): "Estilos sanos de vida en adultos con discapacidad intelectual: qué conocen, factores que los benefician y dificultan”. Revista Síndrome de Down, 30 (I I 6): 20-32.

Davis, J.M. (2008): “Investigaciones y textos etnográficos en el ámbito de los estudios sobre discapacidad”. En Barton, L. (Comp.): Superar las Barreras de la Discapacidad. Madrid: Morata: 34I-358.

Díaz, M.L. et al. (200I): "Taller de comunicación para alumnos gravemente afectados", 200I: Odisea de la Comunicación. Murcia: Sociedad Española de Comunicación Aumentativa y Alternativa: 20I-209.

Echeita, G. y Jiménez, D. (2007): “Un estudio de caso sobre la situación académica, emocional y relacional de alumnos con necesidades educativas especial asociadas a discapacidad intelectual integrados en IES”. Siglo Cero, 222: 17-43.

Edgerton, R.B. (1967): The Cloak of Competence: stigma in the lives of the mentally retarded. Berkeley: University of California Press.

Farmer, M. y Fraser, M. (20I I): Involving Disabled People in Social Research. Londres: Office for Disability Issues (en línea). <http://odi.dwp.gov. uk/docs/res/research/involving-disabled-peoplein-social-research.pdf $>$, acceso I 6 de octubre de 2013 .

Fernández-Cid Enríquez, M. (20I2): Diversidad intelectual. Madrid: Síntesis.

Flores, N. et al. (2010): "Análisis de la calidad de vida laboral en trabajadores con discapacidad”. Zerbitzuan, 47: 95-107.

Flores, R. y Romero, R. (2008): "Las emociones del niño autista a través del comic: estudio de caso". Pixel-Bit 33: 5-28.

Fullana, J. et al. (2003). "La investigación sobre los procesos de integración laboral de personas con discapacidad en entornos ordinarios. Un estudio de casos cualitativo". Revista de Investigación Educativa, 2 I (2): 305-32I.

Gallego, J.L. (2008): "La planificación de la expresión escrita por alumnos con retraso mental”. Revista de Educación 346: 267-290.

Gerber, D.A. (2008): "Escuchar a las personas con discapacidad. El problema de la voz y la 
autoridad en el libro de Robert B. Edgerton The Cloak of Competence”. En Barton,

L. (Comp.): Superar las Barreras de la Discapacidad. Madrid: Morata: 275-298.

Guerrero Muñoz, J. (20I0): “La discapacidad intelectual en el contexto de la investigación etnográfica: rutas y enclaves". Gazeta de Antropología 26(2) (en línea). <http://www.ugr. es/ pwlac/G26_37Joaquin_Guerrero_Munoz. html>, acceso I 6 de octubre de 2013 .

León, A. (2006): Necesidades de las personas con discapacidad intelectual en proceso de envejecimiento. Bilbao: Diputación Foral de Bizkaia.

Morris, J. (2008): "Lo personal y lo político. Una perspectiva feminista sobre la investigación de la discapacidad física”. En Barton, L. (Comp.): Superar las Barreras de la Discapacidad. Madrid: Morata: 31 5-326.

Muñoz, M.T. et al. (2009): "Influencia del lenguaje no verbal (gestos) en la memoria y el aprendizaje de estudiantes con trastornos del desarrollo y discapacidad intelectual: una revisión”. Revista Signos: Estudios de Lingüística, 69: 29-49.

Oliver, M. (2008): “¿Están cambiando las relaciones sociales de la producción investigadora?". En Barton, L. (Comp.): Superar las Barreras de la Discapacidad. Madrid: Morata: 299-3I4.

Oliver, M. (I998): “¿Una sociología de la discapacidad o una sociología discapacitada?”. En Barton, L. (Comp.): Discapacidad y Sociedad. Madrid: Ed. Morata: 34-58.

Ochoa de Eribe, M. (I994): "El lenguaje escrito en la comunicación del paralítico cerebral nooral”. Lenguaje Alternativos para Personas con Dificultades en la Comunicación. España: Ciencias de la Educación preescolar y especial: 79-87.
Ortí, M. y Díaz, E. (20I2): "Claves teóricas y metodológicas para la Investigación Acción Participativa (IAP)". En Arroyo, M. y Sádaba, I. (Coords.): Metodologías de la Investigación Social. Técnicas innovadoras y sus aplicaciones. Madrid: Editorial Síntesis: I 53-I74.

Rodríguez, M.C. (20I0): Vivir sin barreras: la historia de Luigi "Pucho" Maccione contada por su familia y amigos. Madrid: Grupo 5 Acción y Gestión Social.

Rosa, L. (2008): La historia de vida de Ángel: parálisis cerebral, normalidad y comunicación. Madrid: La Muralla.

Santamaría, M. et al. (20I2): "Calidad de vida percibida por los trabajadores con discapacidad intelectual en empleo ordinario". Siglo Cero, 242: 46-6I.

Tamarit, J. y Espejo, L. (20I3): “Experiencias de empoderamiento de personas con discapacidad intelectual o del desarrollo". Siglo Cero, 246:26-39.

Verdugo, M.A. y Rodríguez, A. (2008): "Valoración de la inclusión educativa desde diferentes perspectivas". Siglo Cero, 228: 5-25.

Walmsley, J. (2008): “Normalización, investigación emancipadora e investigación inclusiva en el ámbito de la discapacidad intelectual". En Barton, L. (Comp.): Superar las Barreras de la Discapacidad. Madrid: Morata: 359-380.

Ward, K. y Trigler, J.S. (2002): “Reflexiones sobre la investigación participativa con personas que tienen discapacidades en el desarrollo". Siglo Cero, I99: 45-47.

Welsby, J. y Horsfall, D. (20I I): “Everyday practices of exclusión/inclusión: women who have an intellectual disability speaking for themselves?". Disability \& Society, 26 (7): 795-807.

Williams, V. (I999): "Researching together". British Journal of Learning Disabilities, 27: 48-5I. 